IN PRACTICE

\title{
Symptoms of non-gonococcal urethritis in heterosexual men: a case control study
}

\author{
P Iser, TR H Read, S Tabrizi, C Bradshaw, D Lee, L Horvarth, S Garland, I Denham, C K Fairley
}

Sex Transm Infect 2005;81:163-165. doi: 10.1136/sti.2004.010751

Objective: To determine microbial and behavioural factors contributing to non-gonococcal urethral symptoms in men. Methods: Case-control study of heterosexual men with nongonococcal urethral symptoms (cases) and without urethral symptoms (controls) attending Melbourne Sexual Health Centre, Australia. Sexual behaviour and condom use were measured by questionnaire. First stream urine was tested for potential pathogens: Chlamydia trachomatis (ligase chain reaction), Mycoplasma genitalium (polymerase chain reaction, PCR), Ureaplasma urealyticum (culture and PCR), and Streptococcus spp, Gardnerella vaginalis, and Haemophilus species (culture). Urethral smears from cases were examined for polymorphonuclear leucocytes.

Results: 80 cases and 79 controls were recruited over 4 months in 2002-3. 49 cases (61\%) had urethritis by microscopic criteria, 17 (21\%) had Chlamydia trachomatis (adjusted odds ratio (OR) 27 (95\% confidence interval (CI): 3.4 to 222)), five (6\%) had Mycoplasma genitalium (OR 6.1 (95\% Cl: 0.6 to 61$)$ ), and 11 (14\%) had Gardnerella vaginalis (OR $9.0(95 \% \mathrm{Cl}$ : 1.6 to 52$)$ ). Other organisms were not significantly associated with urethral symptoms. The presence of urethritis on urethral smear did not predict the presence of Chlamydia trachomatis (OR $1.7195 \% \mathrm{Cl}: 0.5$ to 5.4)). Urethral symptoms were significantly associated with unprotected vaginal sex with more than one casual partner (OR 9.3 (95\% Cl: 1.3 to 65)) and unprotected anal sex with a regular partner in the past month (OR $3.5195 \% \mathrm{Cl}$ : 1.0 to 13)).

Conclusion: Gardnerella vaginalis and unprotected anal sex may cause symptoms of non-gonococcal urethritis. Microscopy of the urethral smear to diagnose urethritis in this population does not help to identify which men with urethral symptoms require treatment for chlamydia.

$\mathrm{T}$ he cause of most cases of non-gonococcal urethritis (NGU) remains unknown. Chlamydia trachomatis accounts for $30-40 \%^{12}$ and Mycoplasma genitalium is now known to cause a smaller proportion, ${ }^{2}{ }^{3}$ while other established causes are uncommon. ${ }^{4}$ Ureaplasma urealyticum, Gardnerella vaginalis, Haemophilus species, and Streptococcus spp have been associated with NGU but their causal role is unproved. $^{45}$

In Australian general practice men are often treated when they present with symptoms of NGU before laboratory confirmation of urethritis on urethral smear. It is therefore useful to establish the prevalence of urethral pathogens in all men with urethral symptoms regardless of the presence of urethritis on the urethral smear. We conducted a case-control study of men with symptoms of NGU and controls examining the prevalence of known and suspected pathogens and associations with sexual practices.

\section{METHODS}

The study was conducted at Melbourne Sexual Health Centre, Australia, from December 2002 to March 2003 with ethics committee approval. Men were included if they had urethral symptoms (discharge, dysuria, or urethral discomfort) for less than 1 month. Men were ineligible if they had HIV infection, urethral gonorrhoea on Gram stain, male sexual partners, a current episode of genital herpes, or had taken antibiotics in the previous month. Controls were men without urethral symptoms attending the clinic during the same period, with the same exclusion criteria. Cases had a urethral smear collected and all participants provided a first pass urine specimen and completed a behavioural questionnaire.

Polymorphonuclear leucocytes on Gram stained urethral smear were counted in five oil immersion $\times 1000$ polymorphs per high power fields (phpf). Urethritis was defined as the presence of 5 polymorphs/hpf or more. First stream urine from cases and controls was tested for streptococci, Haemophilus species, Gardnerella vaginalis, and Ureaplasma urealyticum by culture and Chlamydia trachomatis by ligase chain reaction (LCx, Abbott Diagnostics, Abbott Park, IL, USA) and Mycoplasma genitalium and Ureaplasma urealyticum by polymerase chain reaction (PCR).

A volume of $1 \mathrm{ml}$ of urine was centrifuged and resultant pellet extracted using the automated MagNA Pure LC (Roche Molecular Biochemical, Mannheim, Germany) with the DNA Isolation Kit I protocol. Each extracted DNA was amplified by PCR for two targets utilising $5 \mu \mathrm{l}$ aliquots of the DNA. Detection of $M$ genitalium was performed as described previously. ${ }^{6}$ Detection of $U$ urealyticum was performed using adaptation of a previously described assay targeting amplification of a 429 base pair sequence of urease structural gene to real time PCR using a Taqman probe. ${ }^{7}$

Data were analysed on SPSS for Windows Version 11, Chicago, IL, USA. Crude odds ratios and 95\% confidence intervals were calculated for categorical variables and numerical variables were analysed using a $t$ test or nonparametric equivalent. Two adjusted analyses were performed using logistic regression. In the first, all identified organisms were modelled to determine which were independently associated with NGU after adjusting for the primary behavioural risk factor, unprotected vaginal sex, and past urethral symptoms. In the second, odds ratios for the association of selected behavioural characteristics with NGU symptoms were assessed after adjusting for the main risk factor, unprotected vaginal sex. This study had a power of $80 \%$ to detect an odds ratio of 2.6 if the exposure variable was present in $30 \%$ of the controls ( $p=0.05$ two sided). Abbreviations: NGU, non-gonococcal urethritis; PCR, polymerase
chain reaction 
Table 1 Crude and adjusted odds ratios (OR) for organisms and behavioural risk factors

\begin{tabular}{|c|c|c|c|c|c|}
\hline & & Cases (\%) & Controls (\%) & Crude OR $(95 \% \mathrm{Cl})$ & Adjusted $\mathrm{OR}^{*}$ \\
\hline \multicolumn{2}{|l|}{ Number } & 80 & 79 & & \\
\hline \multirow{2}{*}{\multicolumn{2}{|c|}{$\begin{array}{l}\text { Chlamydia trachomatis } \\
\text { Ureaplasma urealyticum (culture or PCR) }\end{array}$}} & $17(21.3)$ & $1(1.3)$ & $21.0(2.7$ to 163$)$ & 27 (3.4 to 222$)$ \\
\hline & & $23(28.8)$ & $26(32.9)$ & $0.8(0.4$ to 1.6$)$ & $0.5(0.3$ to 1.4$)$ \\
\hline \multicolumn{2}{|l|}{ All streptococci } & $28(35.0)$ & $14(17.7)$ & $2.5(1.2$ to 5.2$)$ & $3.2(1.1$ to 9.6$)$ \\
\hline \multicolumn{2}{|l|}{ Group B Streptococcus } & $11(13.8)$ & $5(6.3)$ & $2.4(0.8$ to 7.1$)$ & $2.1(0.5$ to 11.4$)$ \\
\hline \multicolumn{2}{|l|}{ Haemophilus influenzae } & $2(2.5)$ & 0 & $p=0.5$ & \\
\hline \multicolumn{2}{|l|}{ Gardnerella vaginalis } & $11(13.8)$ & $2(2.5)$ & $6.1(1.3$ to 29$)$ & 9.0 (1.6 to 52$)$ \\
\hline \multicolumn{2}{|l|}{ Mycoplasma genitalium } & $5(6)$ & $1(1.3)$ & $4.1(0.5$ to 37$)$ & $6.1(0.6$ to 61$)$ \\
\hline \multicolumn{2}{|l|}{ Previous similar symptoms $\dagger$} & $36(45)$ & $19(24)$ & 2.5 (1.2 to 5.4$)$ & $2.4(1.1$ to 5.4$)$ \\
\hline \multicolumn{2}{|l|}{ Regular sexual partner } & $44(57.1)$ & $48(64.0)$ & $0.8(0.4$ to 1.4$)$ & \\
\hline \multicolumn{2}{|l|}{ Vaginal sex } & 39 (51.3) & $38(51.4)$ & $1.0(0.5$ to 1.9$)$ & \\
\hline \multicolumn{2}{|l|}{ Oral sex } & $36(47.4)$ & $38(51.4)$ & $0.9(0.4$ to 1.6$)$ & \\
\hline \multicolumn{2}{|l|}{ Anal sex } & $11(14.5)$ & $4(5.5)$ & $2.9(0.9$ to 9.6$)$ & $\begin{array}{l}3.5(1.0 \text { to } 13) \\
p=0.05\end{array}$ \\
\hline \multicolumn{2}{|l|}{ Casual sexual partner (past month) } & $45(58.4)$ & $17(23.3)$ & $4.6(2.3$ to 9.4$)$ & \\
\hline \multirow{4}{*}{ Vaginal sex } & 0 & $38(50)$ & $59(68)$ & & 1.0 \\
\hline & 1 & $21(28)$ & $12(16)$ & $1.9(0.9$ to 4.3$)$ & $2.1(0.7$ to 6.1$)$ \\
\hline & $>1$ & $17(22)$ & $2(3)$ & $10.2(2.3$ to 46.1$)$ & $9.3(1.3$ to 65$)$ \\
\hline & & & & & $p$ trend 0.01 \\
\hline \multirow[t]{4}{*}{ Oral sex } & 1 & $18(24.0)$ & $8(11.1)$ & $2.5(1.0$ to 6.3$)$ & $1.8(0.5$ to 6.1$)$ \\
\hline & $>1$ & $16(13.3)$ & $4(5.6)$ & $4.6(1.5$ to 14.6$)$ & $1.7(0.3$ to 8.5$)$ \\
\hline & & & & & $0.7(0.1$ to 3.7$)$ \\
\hline & & & & & $p$ trend 0.15 \\
\hline \multirow[t]{3}{*}{ Anal sex } & Any & $3(4.3)$ & $1(1.4)$ & $3.1(0.3$ to 30.9$)$ & \\
\hline & 1 & $2(2.9)$ & $1(1.4)$ & $2.1(0.2$ to 23.2$)$ & \\
\hline & $>1$ & $1(1.4)$ & & & \\
\hline
\end{tabular}

*The first adjusted analysis included having a casual sexual partner in the previous month, past urethral symptoms, and the presence of other urethral organisms and a second adjusted analysis included anal sex with regular partner and vaginal and oral sex with casual partners.

tRefers to similar symptoms in a separate episode more than 1 month earlier.

\section{RESULTS}

The 79 controls (mean age 30.2 years) were younger than the 80 cases (mean 35.8 years). Forty nine cases (61\%) had 5 polymorphs/hpf or more and 19 (23\%) had fewer than $1 \mathrm{p} /$ hpf. Detection of Chlamydia trachomatis, Streptococcus spp, and Gardnerella vaginalis was statistically associated with urethral symptoms in the crude and adjusted analysis (table 1). When only cases with urethritis were included, both the crude and adjusted odds ratios remained essentially unchanged (data not shown). Mycoplasma genitalium was detected in five cases and one control, but this was not statistically significant.

Among cases the presence of 5 polymorphs/hpf or more in the urethral smear did not predict the presence of Chlamydia (OR 1.7 (95\% CI: 0.5 to 5.4)) or any other organism ( $p>0.15)$. The presence of urethral discharge on history or examination was statistically associated with the detection of chlamydia in cases, OR 3.9 (95\% CI: 1.1 to 15) and 3.9 (95\% CI: 1.2 to 14 ) respectively as was a history of previous NGU symptoms (OR 2.4 (95\% CI 1.1 to 5.4). No other symptoms or signs were associated with the detection of other organisms $(\mathrm{p}>0.2)$.

The behavioural factors associated with NGU are shown in table 1 . In the adjusted analysis unprotected vaginal sex with more than one casual partner and unprotected anal sex with a regular partner were significantly associated with NGU, but unprotected oral sex was not.

\section{DISCUSSION}

In this case-control study of heterosexual men with urethral symptoms, cases were more likely to have Chlamydia trachomatis, Gardnerella vaginalis, and any streptococci isolated from their urine and to report past urethral symptoms, unprotected vaginal sex with a casual partner, and unprotected anal sex with their regular partner. These results are particularly applicable to health services where most men with urethral symptoms are treated in primary care without access to on-site microscopy.

Gardnerella vaginalis was isolated from significantly more cases $(14 \%)$ than controls $(3 \%)$ indicating that organisms associated with bacterial vaginosis (BV) may cause urethral symptoms in men. $G$ vaginalis has been isolated from men with urethritis, ${ }^{5}$ and NGU has also been associated with the presence of $\mathrm{BV}$ in female partners. ${ }^{8}$

In this study unprotected anal sex with a regular partner was reported three times more commonly in cases than controls. It is possible that urethral irritation could result from exposure to rectal flora during anal sex.

Among men with urethral symptoms, urethritis by microscopic criteria did not predict the presence of Chlamydia trachomatis, whereas discharge was predictive. Several studies have also found that microscopic urethritis did not predict $C$ trachomatis, ${ }^{9}{ }^{10}$ while Horner et al found discharge to predict $C$ trachomatis. ${ }^{1}$ The value of urethral symptoms over microscopy in predicting those likely to have $C$ trachomatis, is supported by our finding that the prevalence of $C$ trachomatis among urethritis negative cases (five of 31) was higher than among asymptomatic controls (one of 79; $\mathrm{p}=0.002$ ).

It is possible that our sample size, and the inclusion of cases without microscopic urethritis, prevented us from detecting weaker associations, such as those between individual organisms, microscopic urethritis, or certain behaviours. Ureaplasma urealyticum, Haemophilus influenzae, or any particular streptococcal species were not associated with urethral symptoms.

Acute urethral symptoms in a male population with a low prevalence of bacterial STIs may be due to other causes, including $G$ vaginalis and anal sex. Our results support our practice of offering treatment for $C$ trachomatis and partner notification to all men attending our clinic with symptoms of urethritis and a behavioural risk factor regardless of the presence of urethritis on their urethral smear.

\section{ACKNOWLEDGEMENTS}

We gratefully acknowledge the assistance of Andrew Buchanan, Tina Schmidt, Stella Heley, Helen Henzel, Karen Berzins, Richard Teague, Margaret Morris, Carol Hopkins, Steve Rowles, and Kirsty McNab and the RWH Molecular Microbiology Research Fund. 


\section{CONTRIBUTORS}

PI carried out the research project; TR and CF contributed to protocol design and wrote the paper with CB; ST and SG performed the PCR assays and contributed to the paper; ID and LH contributed to protocol design; and LH performed diagnostic microbiology; DL contributed to the paper.

\section{Authors' affiliations}

P Iser, C Bradshaw, D Lee, C K Fairley, School of Population Health, University of Melbourne, Parkville, 3052, Australia

T R H Read, C Bradshaw, D Lee, L Horvarth, I Denham, C K Fairley, Melbourne Sexual Health Centre, 580 Swanston Street, Carlton, 3053, Australia

S Tabrizi, S Garland, Royal Women's Hospital, Grattan Street, Carlton, 3053, Australia

Conflict of interest. None declared. The study was internally funded.

Ethical approval: The ethics committee of the Victorian Department of Human Services approved this study.

Correspondence to: Christopher K Fairley, Melbourne Sexual Health Centre, 580 Swanston Street, Carlton, Victoria 3053, Australia; cfairley@unimelb.edu.au

Accepted for publication 16 June 2004

\section{REFERENCES}

1 Horner PJ, Thomas B, Gilroy CB, et al. Do all men attending departments of genitourinary medicine need to be screened for non-gonococcal urethritis? Int J STD AIDS 2002;13:667-73.

2 Horner P, Thomas B, Gilroy CB, et al. Role of Mycoplasma genitalium and Ureaplasma urealyticum in acute and chronic nongonococcal urethritis. Clin Infect Dis 2001;32:995-1003.

3 Taylor-Robinson D. Mycoplasma genitalium - an up-date. Int J STD AIDS 2002;13:145-51.

4 Schwartz MA, Hooton TM. Etiology of nongonococcal nonchlamydial urethritis. Dermatol Clin 1998;16:727-33, xi.

5 Lefevre JC, Lepargneur JP, Bauriaud R, et al. Clinical and microbiologic features of urethritis in men in Toulouse, France. Sex Transm Dis $1991 ; 18: 76-9$

6 Yoshida T, Deguchi T, Ito M, et al. Quantitative detection of Mycoplasma genitalium from first-pass urine of men with urethritis and asymptomatic men by real-time PCR. J Clin Microbiol 2002;40:1451-5.

7 Blanchard A, Hentschel J, Duffy L, et al. Detection of Ureaplasma urealyticum by polymerase chain reaction in the urogenital tract of adults, in amniotic fluid, and in the respiratory tract of newborns. Clin Infect Dis

1993;17(Suppl 1):S148-53.

8 Keane FE, Thomas BJ, Whitaker $\mathrm{L}$, et al. An association between nongonococcal urethritis and bacterial vaginosis and the implications for patients and their sexual partners. Genitourin Med 1997;73:373-7.

9 Landis SJ, Stewart IO, Chernesky MA, et al. Value of the gram-stained urethral smear in the management of men with urethritis. Sex Transm Dis $1988 ; 15: 78-84$.

10 Janier M, Lassau F, Casin I, et al. Male urethritis with and without discharge: a clinical and microbiological study. Sex Transm Dis 1995;22:244-52. 